\title{
Géopolitique du savoir et philosophie dans le contexte de l'Institut franco-Chinois de Lyon
}

Marie-Julie MAITRE

\section{(2) OpenEdition}

Journals

Édition électronique

URL : http://journals.openedition.org/transtexts/518

DOI : $10.4000 /$ transtexts.518

ISSN : 2105-2549

Éditeur

Gregory B. Lee

Référence électronique

Marie-Julie MAITRE, « Géopolitique du savoir et philosophie dans le contexte de l'Institut francoChinois de Lyon », Transtext(e)s Transcultures 跨文本跨文化 [En ligne], 9 | 2014, mis en ligne le 19 octobre 2015, consulté le 19 avril 2019. URL : http://journals.openedition.org/transtexts/518 ; DOI $10.4000 /$ transtexts.518

Ce document a été généré automatiquement le 19 avril 2019.

(c) Tous droits réservés 


\title{
Géopolitique du savoir et philosophie dans le contexte de l'Institut franco- Chinois de Lyon
}

\author{
Marie-Julie MAITRE
}

\section{Introduction}

1

Bien que beaucoup d'études aient été menées concernant les étudiants chinois partis étudier la philosophie à l'étranger, tel $\mathrm{Hu}$ Shi aux Etats-Unis au début du $\mathrm{XX}^{\mathrm{e}}$ siècle, encore trop peu se sont penchées sur les étudiants qui sont allés se former en philosophie en France et notamment à l'Institut Franco-chinois de Lyon (IFCL). ${ }^{1}$ De même, l'analyse de la place de cette institution dans la géopolitique du savoir moderne est trop peu explorée. ${ }^{2}$ Cet institut fut l'unique exemple d'une université franco-chinoise implantée hors des frontières de la Chine.

2 Durant plus de 25 ans, cette institution a accueilli 473 étudiants dont un quart a obtenu un doctorat de l'Université de Lyon. Lors de leur retour en Chine, ces étudiants prirent part à la direction du pays et formèrent la nouvelle intelligentsia de la Chine, une élite scientifique moderne, à un moment où la chine interrogeait la valeur de ses connaissances. Beaucoup d'entre eux devinrent écrivains, artistes, chirurgiens, professeurs ou hommes politiques.

Huang Zengyue 黃曾樾 et Yuan Zhuoying 袁擭英 sont deux étudiants chinois qui sont venus étudier en France à l'Institut et qui y ont soutenu un doctorat en philosophie. ${ }^{3}$ Une fois leurs études terminées en France, ils sont retournés en Chine et devinrent tous deux enseignants dans des universités en philosophie et en littérature françaises et chinoises.

L'IFCL est à replacer dans le contexte de la fin du XIX et du début du XX $\mathrm{XX}^{\mathrm{e}}$ siècle. Le savoir occidental et français représentait la modernité pour les intellectuels chinois qui remirent en cause leur propre savoir. On peut entendre la modernité avec l'historien 
Prasenjit Duara comme «un discours qui structure la perception du monde non seulement de manière cognitive à travers les catégories de rationalité et de science, mais aussi par le biais de valeurs telles que le progrès et la laïcité, qui sont souvent inextricablement entrelacées avec les premières catégories. $»^{4}$ Concernant le concept de modernité, nous ne soutenons pas l'approche de la ligne évolutionniste ayant pour fin le modèle civilisationnel euro-américain ni l'équation «modernité = Occident». La géopolitique de la connaissance renvoie à l'organisation du savoir qu'a institué l'Occident depuis l'âge des conquêtes au XVIe siècle et le début du colonialisme. Elle a établi une diffusion du savoir dans un sens unique, celui de l'Occident comme producteur de ce savoir. Ce dernier a le rôle de valider le savoir du reste du monde et diffuse le sien comme étant l'unique savoir scientifique valable.

Le terme d'Occident peut être compris comme une catégorie discursive dont l'usage est problématique et ambiguë tout autant que son pendant géographique imaginaire l'orient, et comporte le risque d'essentialisation. Le spécialiste des études asiatiques Naoki Sakai propose d'éviter cet écueil en le définissant non plus tel une catégorie géographique mais comme une "présence ambiguë et omniprésente d'une certaine domination mondiale dont le sujet peut difficilement être identifiable. $»^{5}$ L'Occident peut renvoyer à une position épistémologique dominante et représenter un point de référence pour les cultures non-occidentales pour construire leur savoir.

6 Cette diffusion du savoir moderne/colonial dans une unique direction s'est manifestée à travers une migration d'idées, de littératures et de concepts depuis l'Europe vers la Chine. La «philosophie» fait partie de ces concepts et disciplines. Elle est généralement entendue comme une activité critique et rationnelle, rendue possible grâce à l'émergence du logos (ou "raison », " pensée », " discours » et «étude ») en Grèce au V siècle avant l'ère commune. La philosophie vise à découvrir la vérité par le questionnement, en utilisant la rationalité et la création de concepts. Cette pratique est devenue une discipline, s'est développée et institutionnalisée en Occident. Partant de cette définition, les philosophes européens du XIX ${ }^{e}$ siècle ont décidé que la Chine n'avait jamais développé cette discipline. ${ }^{6}$

7 Anne Cheng a bien expliqué cette exclusion qui repose sur l'argument que la Chine avait arrêté de penser après l'Antiquité ou après le Moyen-âge, et que l'on ne peut pas parler de philosophie chinoise parmi les contemporains, relayant ainsi la pensée chinoise dans un musée. ${ }^{7}$ Elle explique la raison de ces idées préjudiciables pour la philosophie chinoise par le fait que le label philosophique moderne a été défini au XIXe siècle en Europe et fut validé par les intellectuels chinois au XXe siècle. ${ }^{8} \mathrm{C}^{1}$ est cette idée que cet article va aussi tenter d'éclairer à travers le contexte de l'IFCL en analysant comment les étudiants chinois ont étudié la philosophie en France.

8 L'idée exprimée par Anne Cheng et celle de la diffusion unilatérale de la connaissance intellectuelle sont illustrées avec l'introduction au début du XXe siècle du néologisme zhexue 哲學 provenant du terme japonais tetsugaku forgé par le japonais Nishi Amane (1829-1897). Les intellectuels chinois eurent diverses réactions concernant l'introduction de cette traduction du concept de philosophie occidentale, allant du déni jusqu'à la nécessité d'avoir une philosophie basée sur le modèle européen. Les expressions de "philosophie chinoise » ou de "philosophie occidentale » sont elles-mêmes à critiquer dans le sens où non seulement elles essentialisent les philosophies en question mais dénotent également une idée fausse d'unité des pensées, alors que ces dernières sont plurielles et diverses. 
9 Si la philosophie occidentale fut diffusée en Chine via une expansion moderne qui peut s'analyser comme une sorte de domination culturelle de l'Occident sur le reste du monde, on est en droit de se demander si cette géopolitique du savoir philosophique s'est manifestée dans le contexte de l'IFCL, et si oui comment? De même, on peut se demander

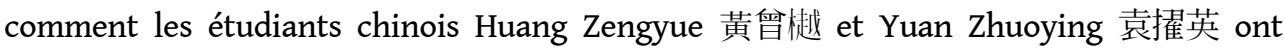
abordé la philosophie européenne et comment ont-ils réagi à cet enseignement.

La littérature existante concernant l'IFCL et son enseignement a concerné son fonctionnement et sa place dans des stratégies d'éducation. Ruth E. S. Hayhoe, dans son article "A Comparative Approach to the Cultural Dynamics of Sino-Western Educational Co-operation" a mentionné l'IFCL dans son examen des systèmes d'éducation sinooccidentaux. ${ }^{9}$ La sinologue visait plus particulièrement à étudier la nature des valeurs académiques créées par les institutions en question en les insérant dans la problématique de la modernisation de la culture académique chinoise ainsi que la manière dont elles furent positives pour la modernisation. ${ }^{10}$ Elle a suggéré que les intellectuels chinois ont admiré le paradigme révolutionnaire et la qualité de la culture académique français qu'ils considéraient comme un héritage mondial. ${ }^{11}$ Hayhoe a décrit l'ethos de l'Institut et son objectif, son fonctionnement, son financement et renseigne plus particulièrement le type de connaissance privilégié qu'on y enseignait tout comme la fonction des étudiants à leur retour en Chine, principalement dans l'éducation supérieure. ${ }^{12}$

Le professeur d'histoire Yann Philippe dans son mémoire de maîtrise L'Institut francochinois un exemple réussi de collaboration en éducation ? a tenté de savoir s'il y a un modèle éducatif spécifique à l'Institut qui aurait tenu compte du mouvement Travail-étude qui l'avait précédé. À travers l'analyse de l'organisation des études à l'Institut, des formations proposées, Yann Phillipe a cherché à savoir si ces formations étaient uniquement scientifiques ou bien également politiques. ${ }^{13}$ L'auteur a démontré notamment que l'Institut a été le fruit d'une relation privilégiée entre Lyon et la Chine, suivant une politique coloniale pour les Français et une modernisation pour les Chinois. Cette analyse aurait pu être replacée dans une perspective postcoloniale afin d'analyser la situation d'un nouveau point de vue, ce que le présent article va tenter de faire.

Florent Villard dans « Lieu d'énonciation, différence culturelle et conscience nationale: la Chine comme objet d'étude dans les thèses des étudiants de l'Institut franco-chinois de Lyon (1921-1946) » a observé simultanément le lieu, la langue et l'objet du discours tenu par les étudiants de l'Institut dans leur thèses et y a cherché les traces d'un discours chinois tout comme une possibilité de spatialiser le terrain où a lieu la production du savoir universalisé de l'épistémologie de la connaissance moderne. ${ }^{14}$ Le spécialiste des études chinoises et transculturelles s'est aussi demandé comment les étudiants ont pu être à la fois sujets connaissant et objets d'étude. Il a étudié les thèses de doctorat des étudiants de l'Institut à la lumière de la théorie de la modernité et de la colonialité de Walter Mignolo. Il a constaté la création d'une situation ambivalente d'énonciation des étudiants, à la fois sujets et objets, "dedans et dehors $» .^{15}$ Florent Villard a en effet soutenu une «impossibilité d'une énonciation hors-sol» puisque les étudiants remplacent le «nous » scientifique neutre et objectif par un sujet collectif « extérieur à la communauté scientifique», idée soutenue par les "paratextes» de leurs travaux scientifiques. ${ }^{16}$

13 Ces analyses sont fondamentales et leurs conclusions précieuses mais l'objectif de cet article est de chercher plus précisément à analyser la place de l'Institut Franco-chinois de Lyon dans la géopolitique du savoir philosophique. Il est question de savoir si on y trouve 
les traces d'une Chine exclue de la philosophie et de la production du savoir et placée comme objet d'étude. Il s'agit aussi de discuter de la place de la philosophie chinoise en France à travers le prisme de l'Institut dans le but d'étendre et combler un manque dans la littérature existante.

Cet article soutient que cette institution est un produit des effets de la géopolitique du savoir intellectuel où le traitement des philosophies occidentales et chinoises les délocalise pour uniquement les temporaliser. Ceci illustre le déplacement de la Chine en tant qu'objet de savoir, anthropos, étudié par les producteurs du savoir, humanitas. Ce mouvement institue l'exclusion de la Chine de la philosophie au moment de l'institutionnalisation de la sinologie. Les théories de l'Orientalisme et du postcolonialisme aident à proposer une réponse qui vise à compléter la littérature existante sur la géopolitique de la connaissance ainsi que sur les échanges intellectuels au début $\mathrm{du} \mathrm{XX}^{\mathrm{e}}$ siècle. La nouvelle approche proposée dans cet article est d'analyser les données existantes de l'IFCL à la lumière des théories postcoloniales et de celles de l'Orientalisme. La méthodologie utilisée consiste à lire les thèses des étudiants sur la philosophie et leurs paratextes à la lumière des théories postcoloniales et de géopolitique du savoir intellectuel.

Il va s'agir en premier lieu de situer l'IFCL à l'intérieur de la géopolitique du savoir en interrogeant le contexte de la fin du XIX ${ }^{\mathrm{e}}$ siècle et du début du XX $\mathrm{XX}^{\mathrm{e}}$ siècle qui éclaire sa raison comme stratégie de modernisation de la Chine et en l'analysant d'un point de vue des théories postcoloniales. Ensuite, il sera question d'identifier la position de la philosophie à l'intérieur de la géopolitique du savoir intellectuel, en questionnant le doute qu'ont éprouvé les intellectuels chinois en l'analysant d'un point de vue postcolonial. Enfin, il s'agira de considérer des points montrant la présence d'un lien entre la philosophie et d'une possible colonialité intellectuelle présentes dans l'IFCL en observant comment furent considérées les philosophies chinoises et européennes par les étudiants mais aussi les professeurs français.

\section{Création de l'Institut Franco-Chinois de Lyon à la lumière de la géopolitique du savoir}

Comment situer l'Institut Franco-Chinois de Lyon à l'intérieur de la géopolitique du savoir dans le contexte de la fin du XIX et du début du XXe siècle? On peut tout d'abord se demander pourquoi l'IFCL a été créé. Pourquoi est-ce que des étudiants chinois furent dépêchés en France pour se former? Pourquoi, sachant que la Chine est détentrice d'un savoir intellectuel, des étudiants en philosophie furent envoyés en France pour en apprendre sa pensée ? On peut trouver une réponse dans le contexte de cette époque où, suite aux deux guerres de l'opium qui ont eu lieu entre 1839 et 1860, la Chine a dû céder des privilèges unilatéraux aux Empires occidentaux lors de différents traités inégaux (Nanjing en 1842 et Tianjin en 1858). Ces traités visaient à imposer à la Chine une " ouverture " qu'elle refusait et a permis aux puissances occidentales d'y exercer une mainmise économique, politique et militaire. Ainsi, la Chine a vu sa souveraineté diminuer dans les domaines de l'administration, de la politique, des finances et de la justice.

17 Le sinologue Chow Tse-Tung a observé deux types de réactions des Chinois face à ces agressions et humiliations répétées. Le premier en réaction à l'impérialisme moderne a 
développé des sentiments patriotiques. Le second, influencé par l'impact de la civilisation occidentale sur la Chine, a suggéré des réformes variées pour la modernisation de la nation. ${ }^{17}$ Il semble que ces événements et la guerre sino-japonaise de 1894-95 aient été la première fois pour les intellectuels chinois d'éprouver un besoin de transformation complète de la civilisation traditionnelle chinoise. ${ }^{18}$ Mais en regardant les résultats de la restauration Meiji au Japon, les jeunes intellectuels chinois ont d'abord pensé qu'il fallait juste additionner à leur savoir l'apprentissage des techniques scientifiques. La Chine devait aussi modeler les lois et institutions politiques sur celles de l'Occident. ${ }^{19}$ En 1898 , les intellectuels pensaient toujours que la philosophie, l'éthique, et les principes fondamentaux de la société traditionnelle chinoise ne devaient pas être changés. Ainsi Zhang Zhidong, 張之洞 (1837-1909) promouvait le principe du ti-yong 體用 les « études chinoises comme structure fondamentale et études occidentales pour usage pratique ${»{ }^{20}}^{20}$

Mais après l'échec des réformes des 100 jours et la révolution de 1911, leur point de vue a radicalement changé et l'occidentalisme a primé chez les intellectuels chinois. En effet, le système du savoir et d'éducation chinois fut remis en cause par les intellectuels car il était fondé sur le système confucianiste incapable de répondre adéquatement aux problèmes du temps selon eux. Ainsi, beaucoup vinrent à penser que la Chine pourrait survivre uniquement si elle adoptait des mesures venant du savoir occidental, un terme qui rassemblait alors les sujets académiques étudiés en Europe, aux États-Unis et au Japon. ${ }^{21}$ Devant l'échec des tentatives de réforme en Chine et à défaut d'une modernisation de l'enseignement, on décida d'envoyer les étudiants chinois étudier à l'étranger, au Japon puis aux États-Unis et en Europe.

Le mouvement d'Éducation à l'étranger liuxue 留學 va concerner plusieurs milliers de jeunes Chinois à partir de la fin du XIX ${ }^{e}$ siècle et jusqu'au début du XXe siècle. Les études à l'étranger avaient comme objectif premier la création d'une intelligentsia nécessaire pour que la Chine accède à la "modernité » désirée et détenue par l'Occident selon les intellectuels occidentalistes. On trouve ainsi chez les intellectuels chinois l'équation " Occident = modernité », que nous allons analyser plus loin. À la suite de l'ouverture des écoles pour apprendre les langues occidentales, on enseigne dès 1866 la technologie occidentale dans des arsenaux à Fuzhou, Shanghai, Suzhou et Nanjing. Des écoles ont lancé un programme de traduction des œuvres occidentales. Y. C. Wang spécifie que comme extension logique, les étudiants furent envoyés étudier à l'étranger pour obtenir les connaissances techniques à leurs sources et revinrent en Chine avec de nouvelles idées. $^{22}$

Parmi les stratégies d'éducation et de modernisation, le mouvement Travail-Études qingong jianxue yundong 勤工儉學運動 suit celui du mouvement d'éducation à l'étranger à partir de la fin du XIX ${ }^{e}$ siècle. Le principe de ce mouvement a été élaboré par Li Shizeng, 李石曾 (1881-1973) qui avait séjourné en France. ${ }^{23}$ Il envisagea le projet de permettre à ses compatriotes de venir étudier en France tout en travaillant pour subvenir à leurs besoins. ${ }^{24}$ Il a créé avec Wu Zhihui, 吳稚暉 (1865-1953) l'Association pour les Études dans la frugalité en France (liufa jianxue hui 留法儉學會), avec l'appui de Cai Yuanpei, 蔡元培 (1868-1940), à l'époque ministre de l'Éducation, et de Wang Jingwei, 汪精卫 (1881-1944), membre de la "Ligue jurée" et proche de Sun Yat-sen, 孫逸仙 (1866-1925). ${ }^{25}$ Une école préparatoire fut ouverte à Pékin, où les étudiants suivent une formation en français et en culture générale durant six mois avec pour objectif de poursuivre leurs études en France. Ce mouvement s'acheva avec la « Marche sur Lyon » de 1921. 
21 L'Institut Franco-Chinois de Lyon fut créé en 1921 suite à ce mouvement et représentait une solution alternative plus stable et plus efficace. ${ }^{26}$ L'objectif annoncé de l'Institut était la formation d'une élite chinoise susceptible de bâtir une nouvelle Chine. Le phénomène sur lequel comptait la Chine était qu'ils transmettraient à leur tour les connaissances et les compétences acquises en France aux jeunes générations de Chinois. Ils pouvaient ainsi contribuer à la réalisation d'un système éducatif modernisé par leur savoir, ainsi qu'à la modernisation des secteurs du pays.

Il semblerait donc que l'IFCL fut une stratégie pour moderniser la Chine en envoyant des étudiants chinois acquérir des connaissances occidentales pour combler le doute concernant le savoir en Chine. Comme le souligne Florent Villard, cette exposition de l'histoire et des relations entre la Chine et l'Occident suit une interprétation téléologique de la « modernisation, européocentrée, évolutionniste et déterministe, où le destin de la Chine, «en retard» et "sous développée», serait de combler son écart sur une civilisation occidentale définie comme « avancée », " moderne » et « industrialisée ». » ${ }^{27}$ Cette notion de modernité fut appliquée à l'Occident et à l'Europe, en tant qu'élément constituant de l'histoire de l'impérialisme européen. Certains intellectuels chinois ont formé cette équation "Occident = modernité ». En effet, l'idéologie de la modernité a développé des notions pour temporaliser le développement humain et le classifier.

23 Ainsi, comme le pense Michael Hardt, le progrès caractériserait l'Europe, l'Occident, et le retard va qualifier les Non-Européens, les Autres et l'Orient. Il a analysé le schéma qui représente les figures des dominés en tant qu'anachroniques, pré-modernes ou primitifs ; et celles des dominants comme modernes. Cette interprétation de l'histoire tient le dominé pour «toujours condamné à l'anachronisme ou à la répétition ; seuls les dominants ont le privilège d'habiter le nouveau et l'actuel. $»^{28}$ Ainsi, la notion de modernité est vue comme un désir du non-occidental ou non-européen et les sociétés non européennes sont représentées comme temporellement mixtes, situées entre tradition et modernité.

Du point de vue de l'IFCL, l'état d'esprit des Français peut être illustré par le discours du ministre de l'Instruction de l'époque, Paul Painlevé. Il a soutenu qu'il fallait «venir en aide à la Chine et non la coloniser. (...) Il importe de collaborer avec les Chinois et non pas d'essayer de les dominer, car le Chinois, veut, à juste titre, qu'on ait de la considération pour lui. $»^{29}$ Le ministre représente ici la position de la France à l'époque des cultures coloniales qui s'étaient dotées de missions civilisatrices. Elles ont ainsi provoqué chez les cultures colonisées ou non-occidentales un doute selon Walter Mignolo :

The map of scholarly production between 1850 and 1945 traced by Wallerstein had scholarship located in Europe and the rest of the World was either the scene of interesting human achievements to study and understand, but frozen in time and antimodern, or the cultures where the civilizing mission had precisely the mission to civilize. The first was the province of civilizational studies (e.g. Orientalism), the second the province of anthropology. The dominant colonial cultures of scholarship were in France, England, and Germany. ${ }^{30}$

L'IFCL pourrait donc être l'une des stratégies pour acquérir le savoir européen afin selon les intellectuels chinois de la fin $\mathrm{XIX}^{\mathrm{e}}$ et du début $\mathrm{XX}^{\mathrm{e}}$ siècle de moderniser leur pays pour faire face aux problèmes de leur temps. Ce doute sur leur propre savoir fut causé par les cultures dominantes dont ils ont observé les méfaits en Chine. Ce doute est à replacer dans une interprétation de l'histoire linéaire et évolutionniste avec comme finalité 
la modernisation de la Chine considérée "en retard», modernité représentée par l'Occident. Ce doute ne concernait pas uniquement les domaines techniques mais aussi intellectuels ce qui est curieux car l'histoire intellectuelle de la Chine n'est pas inexistante.

\section{Philosophie, géopolitique du savoir et Institut Franco- Chinois de Lyon}

Il est question dans ce second mouvement d'identifier la position de la philosophie dans la géopolitique du savoir intellectuel en questionnant le doute qu'ont éprouvés les intellectuels chinois et en l'analysant d'un point de vue postcolonial. Il s'agira aussi d'étudier l'introduction des néologismes et disciplines occidentales telles la philosophie en Chine.

Comme nous l'avons entrevu, certains intellectuels chinois ont remis en cause le système traditionnel et plus particulièrement le système scolaire de la Chine, accusé d'être à l'origine du retard du pays. Ils ont aussi développé une admiration pour le prestige intellectuel, technologique et scientifique de l'Occident. Les années 1903-1906 furent les témoins de l'abolition des examens impériaux et de l'instauration d'un système éducatif «moderne ». Finalement, les années qui suivirent la défaite contre le Japon en 1895 furent principalement caractérisées selon Y. C. Wang par « une adoration aveugle de l'Occident représentant la modernité et par un processus d'imitation sans discernement $»{ }^{31} \mathrm{Ce}$ doute fut inconsciemment entretenu par exemple en France dans le cadre de l'IFCL. Par exemple Jean Bourjade, directeur de thèse de l'étudiant Yuan Zhuoying déclare qu' « exception faite de l'admirable Lao Tseu, la Chine n'offre aucun penseur comparable à nos classiques incontestés : Platon, Aristote.. $»^{32} \mathrm{Ce}$ discours place directement l'étudiant venant de Chine dans une situation de doute de sa propre histoire intellectuelle. Il confirme aussi l'idée dont parlait Anne Cheng que la Chine a arrêté de penser après l'Antiquité ou après le Moyen-âge.

On peut tenter de lire ce doute des institutions et du savoir chinois comme engendré par les cultures d'érudition et de savoir (Cultures of Scholarship) à la lumière de l'analyse du théoricien littéraire Walter Mignolo. ${ }^{33}$ Selon lui, dès le début de l'expansion des cultures européennes dès le $\mathrm{XVI}^{\mathrm{e}}$ siècle, des institutions éducatives furent créées et elles engendrèrent un doute dans les cultures colonisées. Ainsi le savoir qui n'était pas construit via les langues indo-européennes, et même pourrait-on dire selon les structures du savoir occidental, furent mises en doute par les peuples en question. En parallèle du doute de leur propre savoir s'est développée une admiration pour les pays occidentaux (Europe et États-Unis notamment) qui peut s'analyser à partir de la représentation des intellectuels chinois de la France.

Yann Phillipe a analysé le rôle et l'image de la France chez les pensionnaires et cadres chinois de l'IFCL et a conclu que « la France est parvenue à s'imposer comme une sorte de modèle intellectuel $» .^{34}$ Cette position intellectuelle particulière s'explique par des relations étroites entre les deux pays depuis la fin du XIXe siècle ainsi que par plusieurs institutions éducatives installées sur le sol chinois évoquées plus haut. ${ }^{35}$ Ces manifestations confirment l'occidentalisme des intellectuels chinois qui ont ainsi peu à peu érigé la France en une sorte de «modèle de civilisation ». Wang Chung-Hui, ministre des Affaires étrangères de la République de Chine, exprimait cette idée dans un discours : 
31 La France est notre véritable éducatrice dans notre modernisation; nous ne parlons pas seulement ici du machinisme mais bien au sens moral, social et politique. Elle influe et influera sur notre élite, sur nos jeunes générations intellectuelles et cette culture française servira de porte d'entrée à la pénétration économique française. ${ }^{36}$

On peut remarquer la présence de ce phénomène inversé de la Chine représentant la patrie idéale chez les philosophes français du XVII ${ }^{\mathrm{e}}$ siècle, image miroir de la société française absolutiste comme l'a souligné Yann Phillipe. Ainsi la France devint chez les intellectuels chinois une "terre promise» pour la science, la démocratie et la liberté, valeurs centrales du mouvement de la « Nouvelle Culture $» .{ }^{37}$ Ce phénomène où la Chine passe de modèle de civilisation et intellectuel pour l'Europe des Lumières à une nation admirative de la civilisation occidentale est à resituer dans un contexte de circulation des connaissances et sera analysé plus loin. Les Européens furent renseignés sur la Chine et la philosophie chinoise dès le XVI ${ }^{\mathrm{e}}$ siècle par le biais des Jésuites qui fournissaient toutes les informations sur la Chine. Mais qu'en est-il de l'introduction du concept de philosophie européenne en Chine?

La philosophie européenne fut présente en Chine dès l'arrivée des Jésuites. En effet, selon le chercheur en littérature et culture comparée Inaga Shigemi, c'est le Jésuite Julio Aleni (1582-1649) qui aurait en premier fourni une transcription phonétique de "philosophia » feilusuofeiya 斐録所非覀 au XVII ${ }^{\mathrm{e}}$ siècle. ${ }^{38}$ Mais l'introduction du néologisme a eu lieu lors de l'acceptation en Chine en 1890 du terme tetsugaku 哲學 inventé par le Japonais Nishi Amane 西周 (1829-1897) en 1860. Ce terme est une traduction du terme occidental de « philosophie » qu'il a utilisé dans son Hyakuichi shinron de 1874.

Nishi Amane fut envoyé par le gouvernement du Japon à l'Université de Leiden où il a étudié pendant trois ans. Il a traduit de nombreux ouvrages de droit, de philosophie et de science politique et a présenté une vision systématique de la pensée européenne au Japon. ${ }^{39}$ Il est l'une des figures les plus importantes pour la transmission de la littérature scientifique occidentale. Ainsi, aux yeux de cet intellectuel japonais, la philosophie était considérée comme une nouvelle méthode scientifique occidentale plus solide que le confucianisme chinois considéré comme stagnant. Cette discipline fut néanmoins critiquée par un camarade de Nishi en Hollande, Nishimura Shigeki 西村茂樹 (1828-1902) pour son manque de dimension éthique.

35 D’après le philosophe Chen Lai cette introduction a été reprise en Chine, par un diplomate et réformiste chinois, Huang Zunxian 黃遵憲 (1848-1905), qui l'a cité dans son livre en 1890 A History of Japan (Riben guozhi 日本國志), où il a décrit les divers départements des universités au Japon, où une chaire de « Littérature et philosophie chinoises » fut créée en 1881 à l'Université de Tokyo. ${ }^{40} \mathrm{~A}$ partir de 1902, les étudiants chinois au Japon multiplièrent les traductions des essais japonais qui continuèrent d'introduire le terme de philosophie aux intellectuels en Chine. De même, dans la revue Xinmin congbao 新民丵報 éditée par Liang Qichao 梁后超 (1873-1929) à Yokohama paraît, en 1902, un article d'un certain Nai An qui applique le terme sino-japonais à la tradition chinoise. ${ }^{41}$

Le néologisme zhexue 哲學 ne fut pas accepté d'emblée et définitivement par les intellectuels qui utilisèrent d'autres termes pour signifier la philosophie occidentale. Par exemple, le Dictionnaire de la philosophie, compilé par Fan Bingqing 樊炳清 (1877-1929) en 1926, et largement influencé par la terminologie japonaise, ne contient que des termes et expressions de la philosophie occidentale au sens strict. ${ }^{42}$ De même, selon Inaga Shigemi, Yan Fu 㛜復 (1854-1921) dans sa traduction en chinois de l'Évolution et éthique de Thomas 
Huxley de 1898 traduisait le mot «philosophie » par feiluosufei 斐洛看非 et se référait à la terminologie japonaise zhexue pour sa traduction de John Stuart Mill De la liberté en 1903. ${ }^{43}$ Mais c'est enfin avec le Principle of philosophy (zhexue yaoling 哲學要領, 1903) de Cai Yuanpei que s'est consolidée la terminologie du langage chinois. Finalement, le néologisme de philosophie dérivé du terme occidental fut utilisé la première fois pour la philosophie chinoise qui fut catégorisée comme un tout dans la publication Outline of History of Chinese Philosophy (zhongguo zhexue shi dagang 中國哲學史大 綱) de Hu Shi 胡適 (1891-1962), ancien élève de John Dewey, et docteur de l'Université de Columbia. ${ }^{44}$

Le mouvement du savoir philosophique et du concept européen de philosophie est à replacer dans un contexte de flux de connaissances qui correspond à une géopolitique du savoir. À cette époque, la Chine faisait face à l'introduction massive de néologismes venus du japonais, mais qui sont originellement des concepts européens. Selon le sinologue Joël Thoraval, des chercheurs chinois des années 1950 comme Wang Lida ont recensé des centaines de mots venus du japonais et introduits par des anciens étudiants japonais en Occident. Ils appelèrent cela une «nipponisation» (Ribenhua 日本話) du vocabulaire chinois au début du XXe siècle. ${ }^{45}$

Thoraval parle même de double traumatisme car « ces notions occidentales n'ont pu être adoptées qu'au travers d'une médiation japonaise qui, pour des raisons historiques, est souvent vécue sur le mode de la dénégation. $\star^{46}$ On peut également y voir une double influence, premièrement occidentale pour l'origine de la notion, puis japonaise pour le véhicule de la notion. La mise en place d'une influence intellectuelle est bien visible et semble aller bien au-delà de l'introduction d'un terme. Au-delà d'un concept, c'est en effet tout un système avec des normes académiques, un mode de réflexion qui s'impose peu à peu en Chine. Se met en place une « universalisation du paradigme occidental ». ${ }^{47}$

Ce mouvement des idées et des concepts ou disciplines pourrait tenter de s'analyser et de s'expliquer à l'aide des théories postcoloniales et notamment à l'aide du concept de " colonialité ». En effet, si la Chine n'a pas subi de colonisation complète de son territoire par un pays Européen ou Occidental, elle fait néanmoins face à une présence moins évidente, celle du savoir et de la connaissance, des concepts, du système éducatif et des disciplines occidentales. Le sociologue Anibal Quijano appelle cela la «colonialité intellectuelle ${ }^{48}{ }^{48} \mathrm{La}$ colonialité n'aurait pas besoin de la colonisation pour exister, c'est le cas en Chine et au Japon selon Walter Mignolo. ${ }^{49}$ Ramon Grosfoguel rapporte qu'il s'agit d'« un système tissé de formes multiples et hétérogènes, de hiérarchies/dispositifs sexuels, politiques, épistémiques, économiques, spirituels, linguistiques et raciaux de domination et d'exploitation à l'échelle mondiale. ${ }^{50}$ De ce point de vue, la colonialité et la modernité sont les deux faces d'une même médaille. La colonialité est la « continuité de la domination et des formes d'exploitation qui suivent la disparition des administrations coloniales produites par les structures hégémoniques et cultures du système-monde capitaliste/patriarcal moderne/colonial. $\nu^{51}$

Il semble que la colonialité constitue un ensemble de valeurs qui structure une idéologie née avec le monde moderne du $\mathrm{XVI}^{\mathrm{e}}$ siècle (avec la rencontre de l'Amérique par Christophe Colomb et d'autres explorateurs). Il s'agirait d'un principe et d'une stratégie de contrôle qui va au-delà de la simple exploitation économique. La colonialité institue l'eurocentrisme qui devient une source de discriminations religieuses, ethniques et en particulier épistémiques. 
41 Ainsi la colonialité intellectuelle serait illustrée par cette exportation de concepts et disciplines occidentales en Asie via les étudiants qui furent envoyés pour apprendre le savoir occidental

On peut se demander comment l'introduction du concept de philosophie occidentale dans le cadre de la géopolitique du savoir intellectuel a été accueillie par les intellectuels chinois. Même si une occidentalisation de la culture chinoise fut souhaitée par les intellectuels cités ci-dessus, elle suscita un questionnement des intellectuels chinois sur eux-mêmes. Pour Joël Thoraval, du prestige du modèle européen se dégage une angoisse spécifique, qui «s'énonce sur le mode suivant: "avoir ou ne pas avoir» (de philosophie)? $»^{52}$

43 Le sinologue a dressé une classification des réactions qu'ont eues les intellectuels chinois. La première position selon lui est de dire : «Oui, nous avons une philosophie chinoise, et c'est la même que celle des Occidentaux». La seconde position résiderait dans l'affirmation "Oui, nous avons une philosophie, mais c'est une philosophie différente ». La troisième position est de soutenir que «Non, nous n'avons pas de philosophie, mais nous avons autre chose, qui est aussi bien; sinon mieux. " Et enfin la quatrième et dernière soutient que «Non, nous n'avons pas de philosophie, et il nous faut à tout prix nous en doter d'une, sur le modèle occidental. $»^{53}$

La première position est occidentaliste, et consiste à reconnaître "dans sa propre tradition des entreprises comparables à celles que l'on trouve dans l'univers occidental. » ${ }^{54}$ La seconde dénonce un caractère négateur qui fait disparaitre la spécificité chinoise. Thoraval conseille de construire « un concept global et universel de philosophie et, au sein de ce vaste ensemble, de distinguer des orientations, des problématiques, des accents spécifiques : la philosophie occidentale serait ainsi d'inspiration fondamentalement épistémologique, tandis que la philosophie chinoise serait avant tout une métaphysique morale. $\aleph^{55}$ Mais est-ce que construire un concept global de philosophie ne maintiendrait pas finalement une universalisation de la philosophie, donc du paradigme occidental de la philosophie? Ne faudrait-il pas au contraire garder les spécificités de chacun sans universaliser les pratiques de pensées en leur appliquant le terme de philosophie et en se gardant d'établir une supériorité entre les manières de penser? La troisième position évoquée par Thoraval remet en cause le paradigme philosophique alors que la quatrième position est également occidentaliste, et prend acte d'un manque et vise à y remédier en étudiant la tradition européenne.

N'y a-t-il pas d'autres positions possibles à avoir face à l'introduction de ce concept occidental? Ces positions sont toujours prisonnières du cadre de l'opposition Orient/ Occident. Une autre théorie de la réception de la philosophie occidentale par les intellectuels chinois est présentée par Michael Lackner et repose sur une idée de « face à face éternel » qu'il emprunte à Joseph Levenson. Il évoque la division entre les catégories de vue “ intérieure " et " extérieure " (nei wai 内外). Elles opposent d'une manière générale la tradition chinoise à l'apport occidental. ${ }^{56}$

En effet, il semble qu'au début de l'introduction du savoir occidental au milieu du XIX siècle, les intellectuels ont divisé deux types de savoir, le « savoir chinois », zhongxue 中學 et le «savoir occidental ", xixue 西學 ou nouveau savoir, xinxue 新 ${ }_{\mathrm{g}}^{\mathrm{g}} .{ }^{57}$ Michael Lackner rejoint finalement deux positions de Thoraval : la seconde et une partie de la troisième. Lackner souligne que cette expression " pensée chinoise » renvoie à l'idée qu'elle est à la fois indépendante de la tradition européenne et qu'elle s'en distingue de manière 
essentielle ; et elle postule implicitement que la philosophie chinoise est égale, voire supérieure à la pensée occidentale. $\|^{58}$

Les intellectuels chinois ont donc éprouvé un doute concernant leur savoir intellectuel, doute entretenu par exemple par un des directeurs de thèses des étudiants de l'Institut, et qui fut provoqué par la position dominante de la philosophie occidentale, qui fait partie dans la géopolitique du savoir des connaissances présentées comme la norme scientifique du savoir et de la modernité depuis l'expansion coloniale des nations européennes au $\mathrm{XVI}^{\text {e }}$ siècle. La France ainsi a pris la position de la Chine qui était admirée au XVII ${ }^{e}$ siècle par les philosophes des Lumières et fut encensée par les intellectuels chinois qui considéraient son savoir comme en avance et désirable car moderne.

L'introduction des néologismes et disciplines occidentales telles la philosophie occidentale en Chine illustrerait, selon certains penseurs postcoloniaux, une colonialité intellectuelle et une mainmise de la philosophie occidentale qui s'est diffusée et imposée comme unique système d'enseignement et normes académiques de classification. La colonialité a provoqué des discriminations épistémologiques que l'on retrouve non seulement avec le doute éprouvé par les intellectuels chinois concernant leur savoir mais aussi par l'exclusion de la philosophie chinoise du label "philosophie" et nous allons voir comment l'Institut Franco-chinois de Lyon se situe quant à cette idée.

Colonialité intellectuelle et philosophie à l'Institut Franco-chinois de Lyon

Ce dernier mouvement va s'atteler à analyser de potentiels liens entre la philosophie et une colonialité intellectuelle à l'Institut Franco-chinois de Lyon en observant comment furent considérées les philosophies chinoises et européennes par les étudiants mais aussi les professeurs français.

51 On peut tout d'abord s'interroger sur le type de philosophie que les étudiants ont pris pour objet d'étude dans leurs thèses. Huang Zengyue 黃曾樾 a mené une étude des philosophies de Laozi, Kongzi, Mozi alors que Yuan Zhuoying 袁擭英 l'a fait sur la philosophie morale et politique de Mencius. Pourquoi les étudiants en philosophie ont-ils rédigé leurs thèses sur des philosophies chinoises alors que le but de leur séjour fut de se former au savoir européen et français? Du point de vue de la direction pédagogique, il semble que Maurice Courant encourageait les premiers étudiants qui avaient des difficultés en français à étudier des sujets portant sur la culture chinoise. Mais ce n'était pas le cas de Huang Zengyue ni celui de Yuan Zhuoying qui avaient un parfait niveau de français. De plus, Yuan Zhuoying lors de son entrée à l'université de Beijing désirait étudier la philosophie occidentale qui y était absente, il avait alors fini par étudier la littérature occidentale. Il avait donc déjà une base de connaissances sur la culture occidentale lors de son entrée à l'Institut. ${ }^{59}$ Cette attitude de Maurice Courant est contradictoire avec la nécessité de fournir à la Chine des étudiants formés à la science occidentale selon ses besoins de modernisation.

À partir de 1927, les autorités chinoises ont recadré l'orientation du choix des études vers moins de thèses sur les matières chinoises, ces docteurs étant difficiles à placer à leur retour. ${ }^{60}$ On peut aussi comprendre le choix du sujet de thèse des étudiants à travers les déclarations d'Antoine Vicard. Il s'agit pour l'IFCL d'apporter la science occidentale en Chine, au plan des techniques et des idées, de contribuer au développement des études françaises. Mais il s'agit également « de contribuer à une meilleure connaissance de la civilisation chinoise en Occident. En effet, il persiste encore dans les schémas mentaux français l'image, datant du début du XIX ${ }^{\mathrm{e}}$ siècle, d'une Chine attardée et dominée par une 
mentalité de "perpétuelle assistée $"{ }^{61}$ On retrouve cette idée de contribuer à une meilleure connaissance de la Chine et de sa culture chez Jean Bourjade, membre de jury de thèse de Huang Zengyue. ${ }^{62}$

53 Cette thèse de Huang Zengyue sur les trois grands philosophes chinois de l'époque classique Laozi, Konfuzi et Mozi: «nous a appris à l'un et à l'autre, beaucoup de choses que nous ignorions, moi parce que je ne sais pas le chinois, vous parce vous n'êtes pas spécialement philosophe, beaucoup de choses peu connues, quelques-unes peut-être totalement ignorées en Europe. ${ }^{63}$

Mais ce choix d'étude de la philosophie chinoise dans les thèses de ces deux étudiants n'est pas si contradictoire que cela si on le replace dans un contexte de la géopolitique du savoir. En effet, depuis 1814, date à laquelle la première chaire sur la Chine est créée au collège de France, l'étude de la Chine s'est transformée en une pratique professionnelle qui a mené à la naissance de la sinologie. L'institutionnalisation de ce nouveau savoir implique le déplacement de la Chine à une position d'objet d'étude qui n'est plus productrice de connaissance philosophique. La déclaration de Jean Bourjade ci-dessus laisserait entrevoir cette position de la Chine comme objet d'étude, autrement dit selon Walter Mignolo en tant que " partie du reste du monde qui fut le théâtre d'intéressantes réalisations humaines à étudier et à comprendre ${ }^{6.64}$

Il s'agit par l'étude de la Chine de parfaire le savoir philosophique occidental, qui peut être vu comme unique référence du savoir intellectuel. Ainsi, comme le soulignent Anne Cheng et Anne-Lise Dyck, la philosophie chinoise s'est trouvée exclue du champ de la philosophie et nous ajouterons qu'elle fut relayée à celui de la sagesse traditionnelle, non rationnelle..$^{65}$ Anne Cheng a relevé un paradoxe quant au passage de la Chine comme philosophie universitaire à l'objet d'étude de cette nouvelle discipline qu'est la sinologie. ${ }^{66}$ Nous ne sommes pas sûrs que ce soit si paradoxal et il semblerait à nos yeux que ce passage soit plutôt quelque chose de logique si cet événement est resitué dans le contexte de la géopolitique de la connaissance. En effet, comme l'a remarqué Florent Villard, ce passage confirme la géopolitique de la connaissance dans laquelle s'exprime le découpage du savoir qui place les pays occidentaux comme producteurs de ce savoir et les pays du reste du monde comme objets de ce savoir. Nous partageons tout à fait cet avis et nous proposons de pousser l'analyse de cette donnée à la lumière de la théorie postcoloniale en montrant que ce positionnement ancrerait les rôles dans la structure du savoir moderne, positionnement qui serait exprimé à l'Institut Franco-Chinois de Lyon.

En effet, l'exclusion de la Chine comme sujet du savoir et son introduction comme objet de savoir peuvent être analysées selon une lecture postcoloniale à l'aide de la distinction conceptuelle entre antropos et humanitas. Cette distinction renvoie à la différence entre modernité et tradition. ${ }^{67} \mathrm{~L}$ 'antropos est le barbare et l'autochtone tandis que l'humanitas est le moderne et le producteur du savoir. Comme le montre Naoki Sakai,

humanitas a servi à désigner les peuples impliqués dans la production du savoir aussi bien dans la première que dans la deuxième relation, tandis que l'on a progressivement réservé le mot anthropos aux peuples qui ne participent qu'à la première d'entre elles. C'est ainsi que l'humanité dans le sens d'humanitas en est venue à désigner l'humanité européenne, qu'il faut distinguer du reste de l'humanité, pour autant que l'on se fie à l'unité putative de l'Occident et que l'on insiste sur elle. Cela signifie que l'humanité, dans ce sens de l'humanitas, autorise précisément cette distinction de l'Occident de ce que Stuart Hall a désigné en une formule incisive comme « le Reste ». ${ }^{68}$ 
Walter Mignolo souligne que ces concepts permettent à travers ces classificateurs autoritaires d'organiser les catégories de pensées et la production du savoir ainsi que de s'affirmer en disqualifiant ceux qui sont considérés comme déficients du point de vue rationnel et ontologique. Cela est très visible dans le cas de la philosophie chinoise qui est non seulement qualifiée de déficiente ontologiquement (car sa langue est un faible organe de pensée et ne possède pas le verbe être) et rationnellement. ${ }^{69}$ Dans l'histoire intellectuelle européenne par exemple le Jésuite Louis Lecomte voyait dans la langue chinoise une langue complexe, impropre à la science. ${ }^{70}$ Leibniz soulignait à plusieurs reprises "l'impropriété de la langue chinoise à exprimer les choses abstraites, par manque de terme logique et de métaphysique ", cette dernière pouvant être comprise comme la science de l'être en tant qu'être. ${ }^{71}$ Ainsi pour Walter Mignolo, le couple de concepts " modernité » et "humanitas » sont constituant de la civilisation occidentale et cachent une colonialité intellectuelle, cette idée étant à nuancer car simplificatrice et généralisante. Cet ensemble empêche le développement de systèmes de savoirs pluriversaux, dialogiques et démocratiques. Finalement, à l'anthropos comme le souligne Mignolo, on propose l'assimilation ou l'exclusion. ${ }^{72}$

Cette géopolitique de la connaissance intellectuelle serait aussi présente dans la préface d'Edmond Goblot, membre de jury et philosophe, qui soutient que la philosophie chinoise est non seulement autochtone mais aussi traditionaliste. Il déclare que «le traditionalisme est un caractère si profond de la race et de la civilisation chinoises qu'il l'emporte sur la loi de l'évolution des doctrines philosophiques qui ont pris naissance en Chine plus de cinq cent ans avant notre ère, l'une au moins semble n'avoir jamais cessé d'être enseignée dans toutes les écoles de l'immense empire et de former et de nourrir l'âme chinoise comme aujourd'hui encore. $»^{73}$

60 Si on analyse ces déclarations d'après les théories postcoloniales, on retrouverait ici le discours qui selon l'historienne Sophie Bessis soutient que « la tradition est autochtone et la modernité occidentale. $\aleph^{74}$ Dans ce discours, c'est la maturité qui caractérise l'Europe. Elle « se dit « vieille » depuis qu'elle s'est persuadée de l'antériorité de sa civilisation et a annexé de «nouveaux» mondes. $»^{75}$ Ainsi, l'âge adulte et la maturité caractérisent l'Europe alors que l'enfance et la jeunesse définissent l'Orient et la Non-Europe. ${ }^{76}$ Jean Bourjade dans la préface de la thèse de Yuan Zhuoying parle ainsi de la " jeune Chine ${ }^{77}$ Ceci est pourtant en contradiction avec l'idée de civilisation traditionaliste qui selon Edmond Goblot « a les regards tournés vers le passé. Confucius déjà, il y a vingt siècles, ne prétendait pas innover, mais recueillir des traditions plus anciennes encore. La Chine est donc caractérisée à la fois, jeune pour son « immaturité supposée par les producteurs de ce discours, et vieille, tournée vers le passé et retardataire. ${{ }^{78}}^{7 l}$ l'oppose à la civilisation occidentale, qui « progresse par la science, regarde vers l'avenir. ${ }^{79}$ Il ne pense pas que la France au début du $\mathrm{XX}^{\mathrm{e}}$ siècle soit encore civilisée mais que le seront seulement ses descendants. Ainsi, la France doit encore "progresser» vers la civilisation du progrès selon les caractéristiques de ce type de discours évolutionniste. La Chine est donc à la fois décrite comme vieille, traditionaliste et jeune, dans tous les cas, immature.

61 En plus de la dichotomie traditionalisme/modernité qui constitue la géopolitique de la connaissance, on trouve aussi dans les paratextes et les thèses de l'IFCL l'idée que $l^{\prime}$ Europe est le centre du savoir, c'est-à-dire une notion $\mathrm{d}^{\prime}$ " eurocentrisme ${ }^{80}{ }^{80}$ Edmond Goblot illustre cet eurocentrisme du savoir intellectuel qui a guidé Huang Zengyue dans ses recherches et études. Il déclare dans la préface que même si la civilisation chinoise est admirable parce qu'elle n'a pas perdu son savoir philosophique comme les Occidentaux 
ont perdu la philosophie grecque, néanmoins « de la philosophie grecque est issue toute la science humaine", et que "la Chine ait eu aussi sa philosophie autochtone ${ }^{81} \mathrm{Il}$ reconnaît une philosophie propre à la Chine même si à ses yeux, toute la science humaine, ou la science universelle, dérive de la philosophie grecque et donc de l'Europe. Il souligne clairement que la science « invention du génie grec ... manquait à la civilisation chinoise » ${ }^{82}$ et il rappelle l'objectif de son étudiant, être venu « chercher la science en Europe pour l'introduire dans son pays $»{ }^{83}$

62 L'idée d'eurocentrisme dans la pensée pourrait être liée à celle d'universalité, idée défendue par Huang Zengyue qui a évoqué la présence des philosophes chinois dans le monde, et qui de ce fait les a placés au même niveau que les penseurs européens. Il a parlé de la Chine, « fière d'avoir vu naître quelques-uns des plus grands philosophes au monde. Ce sont eux qui ont infusé dans le sang des quatre cents millions d'hommes qui peuplent notre immense pays les sentiments de justice, d'altruisme, de pacifisme dont s'honore notre vieille civilisation. $»^{84} \mathrm{Si}$ l'amorce de sa préface est teintée d'un certain patriotisme, il en résulte néanmoins que cet étudiant pense les philosophes chinois sur la même échelle que les philosophes européens. Ce qui lie ces penseurs est la raison, commune à tous les hommes. En effet, Huang déclare que ce qui constitue le confucianisme, «c'est la raison, la raison pure, la raison humaine, la raison pratique, la raison cosmopolite, la raison sous une forme tellement modeste qu'on pourrait l'appeler bon sens! $»^{85}$ Cette raison est le fond de la doctrine de Confucius, elle est amour de la justice et sentiment du droit.

63 Ainsi pour Huang, ce qui constitue l'héritage des Chinois est la raison et la justice. ${ }^{86}$ Huang reprend l'idée de la déclaration de Descartes du Discours de la méthode (1637) qui fait du bon sens « la chose du monde la mieux partagée », ne la séparant pas de la raison. Ainsi, pour lui, ce qui vient de l'Europe lui sert non seulement une «garantie de sécurité et de confort » mais aussi et surtout « la confirmation de la vérité intellectuelle qui nous prouve que la raison est la chose humaine la mieux partagée $»{ }^{87}$ Huang viserait-il une universalité de la raison qui autoriserait ce traitement égal des philosophes occidentaux et chinois? Il concède une admiration pour l'Occident mais marque bien ce trait d'union entre les hommes :

64 Ainsi, non seulement la grandeur de la puissance mécanique nous fascine, la beauté des magnifiques découvertes nous enivre, mais nous admirons aussi la synthèse méthodique, la dialectique logique, l'esprit scientifique des penseurs occidentaux ; car nous retrouvons sous le himation d'Aristote, sous la toge de Cicéron, sous le pourpoint de Descartes la même raison que nous trouvons sous la robe de Confucius. ${ }^{88}$

Pourtant ce même Descartes déclare que même si la raison est présente tout entière en chaque homme dans l'intelligence, c'est une chose de disposer d'une capacité, c'en est une autre que de l'exercer avec méthode. Et l'exercice méthodique est la raison ellemême, en tant qu'elle est la conduite ordonnée de la pensée. Ainsi, cet argument de la "pensée cosmopolite» s'expose au contre argument de la méthodologie. Cette méthodologie c'est la "synthèse méthodique, la dialectique logique, l'esprit scientifique des penseurs occidentaux » que Huang admire..$^{89}$ De même, il a remarqué que la raison pouvait être employée à diverses finalités comme le soutient P.-L. Couchoud qu'il cite, à savoir que l'Europe a appliqué la raison à la connaissance alors que l'Asie l'avait appliquée aux relations entre les hommes et au perfectionnement de la justice et du bonheur. ${ }^{90}$

À son retour en Chine en 1925, Huang Zengyue continua de délivrer un message d'universalité de la pensée. Il a travaillé en tant qu'ingénieur au Bureau technique de 
Chemin de fer à Beijing. Il fut par la suite nommé à l'Université normale de Beijing comme professeur de littérature française et étudie la poésie chinoise. Il publie aussi des articles sur la philosophie dans un journal dont le nom n'est pas précisé :

67 J'apprends à mes compatriotes que les grands penseurs sont parents, et que rien ne sépare plus les hommes lorsqu'ils arrivent sur les cimes sereines de la pensée. Mais on n'aurait jamais cru que la parenté puisse aller si loin que je le démontre dans ma thèse. Les articles de journal que j'ai fait parfois depuis mon arrivée permettent à certaines classes de mes compatriotes de se rendre compte que nos plus grandes idées, celles auxquelles nous tenons le plus, ont été partagées par vos philosophes et appréciées par vos compatriotes. ${ }^{91}$

Huang suit en quelque sorte la première position qu'exposait Thoraval, les deux philosophies, occidentale et chinoise, ont des caractères et une entreprise qui se recoupent. Il a diffusé ce que représentait l'Institut comme le souligne Mei Duanmu, à savoir un siècle de rêves et d'amitié entre les intellectuels des deux pays que sont la France et la Chine. ${ }^{92}$

69 Une pensée humaniste et une universalité de la pensée se dessinent dans les idées de Huang Zengyue mais d'un point de vue postcolonial, l'universalisme peut être vu comme une autre forme de colonialité intellectuelle. La raison est souvent placée comme point de mesure de la pensée. Cet argument relève de la «différence coloniale» selon Walter Mignolo. En effet, afin de défendre leur position privilégiée en tant que détenteurs de la connaissance et de la "philosophie», certains philosophes européens ont créé la " différence coloniale ", c'est-à-dire qu'ils mettent en évidence ce que la Chine n'a pas (la pensée rationnelle, la science, une écriture abstraite) afin de nier son statut philosophique. ${ }^{93}$

70 Elle est utilisée par des penseurs contemporains français. Alain Badiou par exemple affirme en parlant du travail du sinologue et philosophe François Jullien, que « quand on lisait, avant Jullien, ces textes classiques, sans la préparation et sans le travail conceptuel, on avait l'impression qu'il s'agissait - comment dire ? - de banalités. C'est d'ailleurs ce que Hegel disait à propos de la pensée chinoise. ${ }^{94}$ Ainsi, même si la raison et le bon sens sont partagés, cela ne signifie pas aux yeux des intellectuels occidentaux que la méthodologie pour penser est également partagée. Ils oublient cependant qu'il n'y pas qu'une seule méthodologie pour penser, il y en a autant que d'espaces et de cultures.

71 Les notions de raison et de modernité sont liées à celle de progrès et tissent le discours de la géopolitique de la connaissance. Huang soutient que le progrès implique la concurrence, et la concurrence implique la rivalité. Il reprend une idée que les Jésuites avaient utilisée pour expliquer le manque de progrès scientifique en Chine. En effet, les Jésuites au XVI ${ }^{\mathrm{e}}$ siècle ont décrit les Chinois comme faibles dans les sciences et ont tenté des explications de cette faiblesse. Le Jésuite Parrenin a énuméré deux raisons de ce manque de progrès dans les sciences. La première est que les Chinois «n'ont point de récompense à attendre ${ }^{95}$ et qu'il "n'y a rien ni au-dehors, ni au-dedans qui pique et entretienne l'émulation $»{ }^{96}$ Les Chinois selon lui ont cessé leurs recherches scientifiques, non seulement parce que rien ne les pousse à continuer, mais aussi parce qu'il n'y avait pas de concurrence dans leur recherche de l'intérieur ou sans la Chine. ${ }^{97}$

72 Reste à savoir si ces idées furent reprises par les professeurs français dont les cours et conseils furent suivis par Huang Zengyue. Ainsi, contrairement aux intellectuels occidentalistes qui en Chine accusaient le confucianisme d'être à l'origine du dit 
« retard » de leur pays, Huang cherche à démontrer que Confucius et sa doctrine ne sont pas à son origine. ${ }^{98}$ Huang pense comme Cai Yuanpei que ce ne sont pas la doctrine et les idées du Confucianisme qui sont à rejeter mais plutôt les institutions qui en ont émergées. 99

Un dernier aspect de la géopolitique du savoir intellectuel que l'on pourrait trouver dans la thèse de Yuan Zhuoying est à lier avec le comparatisme en philosophie. ${ }^{100}$ Même si il a choisi de traiter un sujet de philosophie chinoise, Yuan Zhuoying y a mêlé des comparaisons avec les philosophes occidentaux. En effet, après avoir signalé dans son avant-propos l'espoir que sa thèse puisse être une introduction à la philosophie confucéenne ; il a dressé dans le chapitre VII les préceptes d'éducation de Mencius (372289 AEC) tout en les rapprochant de ceux de Jean-Jacques Rousseau (1712-1778).

Yuan a cité ce dernier qui déclarait dans L'Emile (1762) que «tout est bien sortant des mains de l'auteur des choses $"{ }^{101}$ Yuan Zhuoying pense que cette idée fait écho à Mencius, " une voix chinoise antique qui déclarait déjà que notre nature et notre cœur venant du ciel sont primitivement bons. $»^{102}$ Lors d'une analyse comparative, Yuan a dégagé un point commun chez les deux penseurs. Tous deux ont pensé une pédagogie négative qui avait pour mission de protéger l'enfant des mauvaises influences. L'élément principal de la philosophie de l'éducation de Rousseau était de laisser les tendances naturelles de l'enfant s'exprimer de manière libre, sans réprimer ni accélérer sa croissance. La pédagogie de Mencius résidait dans l'adaptation des enseignements du sage aux capacités des élèves ainsi que de laisser les facultés de l'enfant se développer spontanément. ${ }^{103}$

Yuan semble donc construire des ponts entre les deux philosophes séparés dans le temps et l'espace. Mais en termes de comparatisme, les philosophes occidentaux sont le point de référence qu'il utilise. En effet, lorsqu'il veut comparer l'enseignement de Mencius, après Rousseau, il prend Socrate comme point de comparaison : « sa manière d'enseigner est la manière de Socrate $»^{104}$ et " comme Socrate, Mencius considère la conversation comme un art $» .^{105}$ De même, Confucius avance que la science profonde est de connaître les hommes tout comme Socrate qui prônait le « connais-toi toi-même ». ${ }^{106}$

76 Faut-il y voir l'influence des professeurs qui avaient tendance à avoir une vision eurocentrée de la pensée ? Ce n'est pas certain. Par exemple, Jean Bourjade dans la préface de la thèse de Yuan Zhuoying exprime une grande ouverture d'esprit. Il reconnaît "qu'en dépit des modes d'exotisme soit extérieur et pittoresque, soit même intérieur et psychologique, qui ne provoquent jamais que des engouements passagers, la pensée et l'âme de la Chine n'ont généralement occupé que les régions marginales de notre champ d'attention. $\aleph^{107}$ Il remarque le manque d'attention pour la Chine et sa pensée, tout comme sa mise en marge de la philosophie. Parlant du pragmatisme de la philosophie chinoise il interroge: "oserons-nous dire que c'est là une infériorité pour la pensée chinoise ?».108 Cette attitude est intéressante à une époque où on sait que c'est ce pragmatisme et le défaut de développement des sciences spéculatives qui depuis les Jésuites invalident la position de la philosophie chinoise en tant que philosophie comme acte d'abstraction. ${ }^{109}$

77 Ce problème du comparatisme peut encore se lire chez certains auteurs de nos jours. On y trouve les philosophies occidentale et chinoise comme étant diamétralement différentes, relevant d'autres mondes. On peut lire dans la préface de Jean Bourjade que «l'histoire des idées en Chine n'est pas l'histoire des systèmes métaphysiques ontologiques, mais c'est bien plutôt l'histoire de la sagesse et de l'apostolat moral. » ${ }^{110}$ Ainsi on voit se creuser une différence ontologique entre philosophie occidentale et philosophie chinoise, 
la première étant caractérisée par la métaphysique ontologique et la seconde par la sagesse. On peut retrouver cette différence dans une approche de la philosophie chinoise chez François Jullien qui érige la pensée chinoise en tant que fondamentalement autre que la pensée européenne, et qui aide cette dernière à combler ses failles. La pensée chinoise est ainsi vue telle une réalité figée, une pensée ancienne qu'il met à distance pour mieux penser la philosophie européenne. Cette méthode constitue à nos yeux l'expression d'un certain orientalisme intellectuel. ${ }^{111}$ Jean Bourjade l'a formulé bien avant lui : «En Chine, soit dit sans malice pour nous Européens, les philosophes ne sont que des sages, mais le sont, ou veulent l'être, pleinement. $»^{112}$ Faut-il noter une impression de manque dans le «que » de la nature des philosophes chinois : ils ne sont que des sages, et les autres, seraient-ils plus que des sages?

Jean Bourjade caractérise la philosophie chinoise de sagesse chinoise ou de pensée pratique qui lie la raison et la vie. Il s'agit pour lui d'un humanisme intuitif. ${ }^{113}$ Ainsi, Edmond Goblot met en garde Huang Zengyue dans la préface de sa thèse en soulignant qu'avant de venir chercher la science, pour la pratiquer en Chine par la suite, il faut néanmoins "maîtriser la théorie » et même aller plus loin que seulement la connaître mais aussi remonter à ses principes. ${ }^{114} A$ Ainsi, pour devenir savant, conseille Edmond Goblot, il faut acquérir l'esprit scientifique et se faire une âme de savant.

\section{Conclusion} s'était manifestée dans le contexte de l'institut Franco-Chinois de Lyon, et si oui comment. Nous pouvons conclure qu'à travers l'analyse des paratextes et des thèses de deux étudiants en philosophie de l'IFCL, il est apparu que cette institution serait un produit des effets de la géopolitique du savoir intellectuel où le traitement des philosophies occidentales et chinoises suit le découpage moderne du savoir. On y trouverait l'illustration du déplacement de la Chine en tant qu'objet de savoir (anthropos) étudiée par les producteurs du savoir (humanitas), ce qui a institué la Chine en dehors de la philosophie.

81 L'IFCL s'est situé comme faisant partie des stratégies pour acquérir le savoir européen afin, selon les intellectuels chinois de la fin XIX et du début XX ${ }^{e}$ siècle, de moderniser leur pays pour faire face aux problèmes de leur temps. Ce doute sur leur propre savoir fut causé par les cultures dominantes dont ils ont observé les méfaits en Chine. Ce doute est à replacer dans un contexte postcolonial, dans le sens où il suit une interprétation linéaire et évolutionniste de l'histoire avec comme finalité la modernisation - représentée par l'Europe et les États-Unis - de la Chine considérée comme " en retard ».

Le doute éprouvé par les intellectuels chinois concernant leur savoir intellectuel est visible dans les propos d'un professeur de l'IFCL. Il fut provoqué par la position dominante de la philosophie occidentale qui fait partie, dans la géopolitique du savoir, des connaissances présentées comme la norme scientifique de la modernité. La France a ainsi pris la position de la Chine, qui était admirée au XVII ${ }^{\mathrm{e}}$ siècle par les philosophes des Lumières, et fut encensée par les intellectuels chinois qui considéraient son savoir comme en avance et désirable car moderne. L'introduction des néologismes et disciplines occidentales telles la philosophie européenne en Chine illustre la colonialité intellectuelle 
et une mainmise de la philosophie occidentale qui s'est diffusée et imposée comme système d'enseignement et de normes académiques de classification. La colonialité intellectuelle a non seulement imposé un modèle eurocentrique en philosophie, mais elle a aussi provoqué des discriminations épistémologiques que l'on retrouve avec le doute éprouvé par les intellectuels chinois concernant leur savoir et l'exclusion de la philosophie chinoise du label "philosophie".

Des liens entre la philosophie et cette colonialité intellectuelle au sein de l'IFCL furent également mis à jour. Les étudiants Huang et Yuan ont étudié la philosophie chinoise dans leurs thèses non pas parce que leur niveau de français était mauvais mais parce qu'étudier la Chine et sa pensée était approprié pour des natifs. De même, les penseurs étudiés sont des penseurs chinois "antiques" et on retrouve bien l'idée de muséification de la pensée chinoise identifiée par Anne Cheng et entretenue de nos jours par un courant d'étude de la pensée chinoise qu'on peut trouver dans les écrits de François Jullien. On lit dans les thèses, notamment celle de Yuan Zhuoying, un comparatisme utilisant comme point de référence et d'autorité les penseurs occidentaux, auxquels il essaie de rapprocher les doctrines des penseurs chinois afin de les éclairer ou de les valider.

Cette idée que la Chine se retrouve dans une position d'objet d'étude illustrerait une géopolitique coloniale de la connaissance. L'IFCL représente ce passage de la Chine réduite à un objet d'étude (anthropos) et non pas comme productrice de savoir (humanitas ), statut réservé aux occidentaux détenteurs du savoir et du label "philosophie". L'orientalisme et la colonialité se trouvent dans le cadre des paratextes et des thèses de Huang et Yuan, à travers un schéma tradition/modernité érigé pour les civilisations chinoises/européennes et des conceptions eurocentrées et orientalistes du savoir. On y lit une conception universaliste du savoir, mais ce qui est considéré comme universel est le savoir occidental, envisagé comme délocalisé et global. Egalement temporalisé, ce savoir occidental désigné comme moderne est érigé comme la fin ultime de la ligne chronologique du progrès.

Ainsi, on trouverait au sein de l'IFCL la différence coloniale dont parle Mignolo. Elle met en relief ce qu'il manque à la culture non-euro-américaine. Dans le cas de notre étude, elle caractériserait la philosophie chinoise comme déficiente ontologiquement et rationnellement. L'Occident organiserait le savoir en instituant des labels épistémologiques. Le rôle ambiguë de la raison fut aussi observé dans les thèses, raison admirée par les étudiants mais qui peut aussi devenir dangereuse et totalisante si elle est excluante et discriminatoire.

Nous espérons que cet article pourra offrir une nouvelle lecture de cette institution du point de vue de l'organisation du savoir global/moderne, et plus particulièrement du savoir philosophique. On y a trouvé l'expression d'une globalisation de la philosophie occidentale, qui, dans les faits, pourrait apparaitre comme une pensée parmi d'autres si elle était localisée. Elle ne serait donc pas la forme standard et référentielle des pensées de l'humanité mais l'une d'entre elles. 


\section{NOTES}

1. Voir Chou Min-chih, Hu Shih and intellectual choice in modern China. Ann Arbor, University of Michigan Press, 1984, Jerome B. Grieder, $\mathrm{Hu}$ Shih and the Chinese renaissance: liberalism in the Chinese revolution, 1917-1937, Cambridge, Harvard University Press, 1970 et $\mathrm{Hu}$ Shih, The Chinese renaissance: the Haskell lectures, 1933, Chicago, University of Chicago Press, 1934.

2. Voir Florent Villard, « Lieu d'énonciation, différence culturelle et conscience nationale: la Chine comme objet d'étude dans les thèses des étudiants de l'Institut franco-chinois de Lyon (1921-1946) ", Langues, littératures et cultures francochinoises du XXI ${ }^{\mathrm{e}}$ siècle, Taipei, Symposium International 2010, Presses de l'Université Tamkang, 2011.

3. Huang Zengyue 黃曾樾 (1898-1966) est resté pendant un an à l'Institut Francochinois de Lyon de décembre 1924 à octobre 1925 mais était en France depuis février 1921. Ancien élève de l'école du génie maritime de Amoy, Hontcheou, diplômé de l'École centrale de Lyon, cet ingénieur a soutenu une thèse le 27 juin 1925 qui a pour titre l'Étude comparative sur les philosophies de Laozi, Kongzi, Mozi (Lyon, Rey, 1925). Yuan Zhuoying 袁擭英 (1896-1979) fut présent à l'Institut d'octobre 1921 à novembre 1929. Il a soutenu le 23 juin 1927 une thèse intitulée La philosophie morale et politique de Mencius sous la direction de Jean Bourjade (Paris, Geuthner, 1927).

4. "a discourse which structure the perception of the world not only cognitively through the categories of rationality and science, but also by means of such values as progress and secularism, which are often inseparably entwined with the former.", Prasenjit Duara, "Knowledge and Power in the Discourse of Modernity: The Campaigns Against Popular Religion in Early Twentieth-Century China", The Journal of Asian Studies, vol. 50, n 1, February 1991, p. 67.

5. it is an ambiguous and ubiquitous presence of a certain global domination whose subject can hardly be identifiable ", Naoki Sakai, Translation and Subjectivity : On Japan and Cultural Nationalism, Minneapolis, University of Minnesota Press, 1997, p. 61.

6. André Lalande, Vocabulaire technique et critique de la philosophie (volume 2), Paris, Presses Universitaires de France, 1999, p. 774 et Christian Godin, Dictionnaire de philosophie, Paris, Fayard, 2004, pp. 742 et 979.

7. "China stopped thinking after antiquity, or at best after the Middle Ages, and that there is no Chinese philosophy to speak of ever since, not to say among our contemporaries, which is tantamount to confining Chinese thought to the museum.", Anne Cheng, "The Problem with "Chinese Philosophy" ", Revue internationale de philosophie, $n^{\circ} 232,2 / 2005$, p. 176.

8. "the modern philosophical label was defined in the context of Enlightenment Europe, above all in the 19th century, and subsequently claimed by the Chinese intelligentsia at the turn of the 20th century.", Ibid.

9. The China Quarterly, $\mathrm{n}^{\circ} 104$, December 1985, Cambridge University Press on behalf of the School of Oriental and African Studies, pp. 676-699.

10. Ibid., p. 676.

11. Ibid., pp. 688-89.

12. Ibid., p. 696.

13. Lyon, 1998, 2 vol. (Mémoire de maîtrise Université Lumière Lyon II, juin 1998).

14. Langues, littératures et cultures franco-chinoises du XXI ${ }^{e}$ siècle, Taipei, Symposium International 2010, Presses de l'Université Tamkang, 2011.

15. Ibid., p. 50. 
16. Ibid., p. 51.

17. Chow Tse-Tung, The May Fourth Movement: Intellectual Revolution in Modern China. Cambridge, MA: Harvard University Press, 1960, p. 19.

18. Avant la Chine n'avait jamais connue de défi par une quelconque influence étrangère mis à part le bouddhisme indien. Ibid., p. 13.

19. Ibid.

20. Ibid.

21. Timothy Weston, «The Founding of the Imperial University » in Rebecca E. Karl \& Peter Zarrow (Dir.) Rethinking the 1898 Reform Period: Political and Cultural Change in Late Qing China, Cambridge, Harvard University Asia Center, 2002, p. 102. C'est avec le mouvement du 4 mai 1919 que les intellectuels chinois prirent parti de s'inspirer de l'Occident, non seulement dans la technologie, les lois et les institutions politiques mais aussi pour la philosophie, l'éthique, les sciences naturelles, les théories sociales et les institutions, voir Chow Tse-Tung, Ibid., p. 14.

22. Wang Y. C., Chinese Intellectuals and the West 1872-1949, The University of North Carolina Press, 1966, p. 42. Beaucoup d'étudiants revenus de l'étranger ne trouvèrent pas de travail, apparemment parce qu'ils ne voulaient pas se lancer dans les affaires ou commencer en bas de l'échelle. Ils trouvaient également une forte hostilité des fonctionnaires envers eux. John Dewey écrit à leur sujet: “They have been idealizing their native land at the same time that they have got Americanized without knowing it. They have been told that they are the future saviours of their country and then their country doesn't want them for anything at all.», John Dewey, Letters from China and Japan, Evelyn Dewey (Dir.), New York, 1921, p. 190 et p. 244 in Nancy F. Sizer, « John Dewey's Ideas In China 1919 To 1921 »,Comparative Education Review, vol. 10, n³, October 1966, p. 392.

23. Li Shizeng (Yuying) 李石曾 est une autre figure parmi les intellectuels chinois prônant une ouverture à l'égard de l'Occident. Il part en 1903 à Paris, et suit des cours à l'école pratique d'agriculture de Montargis et à l'université de la Sorbonne et à l'Institut Pasteur. Il a développé une morale politique universaliste, syncrétisme entre la tradition chinoise et la pensée libertaire occidentale.

24. L'avantage en France selon Shu Hsingcheng était qu'un an de travail payait deux ans d'études. Shu Hsingcheng Chintai chungkuo liuxieshi 近代中國留學史 Shanghai, 1927, p. 89.

25. Cai Yuanpei 蔡元培 (1867-1940), empreint d'une éducation traditionnelle basée sur la connaissance des classiques, devint jinshi 进士 (docteur) en 1890 après avoir passé les examens impériaux. Témoin des diverses défaites de la Chine face aux pays Occidentaux et au Japon à la fin du XIX siècle, il traduit plusieurs ouvrages européens dans le but d'élucider les raisons de cette prédominance. Il mesure la faiblesse du système scolaire chinois. Il poursuit des études de philosophie, d'esthétique et de psychologie en Allemagne et écrit des manuels d'enseignement de la morale. Il devint ministre de l'Éducation en 1911.

26. Le choix de Lyon s'explique quant à ses relations nouées tôt avec la Chine. Lyon fut considérée comme le point le plus occidental de la Route de la soie, la ville a imprimé les textes des missionnaires sur la Chine, au XVIII ${ }^{\mathrm{e}}$ siècle les soyeux représentaient ce lien. En 1900 la langue chinoise y était déjà enseignée et en 1913 son Université créait une chaire de chinois.

27. Florent Villard, op. cit., p. 2.

28. Michael Hardt, «L'histoire eurocentrée, sur Dipesh Chakrabarty Provincializing Europe ", Multitudes, $n^{\circ} 6,2001 / 3$, p. 37.

29. "Les impressions de M Paul Painlevé retour de Chine", Le Petit Parisien, 29/10/1920.

30. Walter Mignolo, Global Histories, Local Design, Princeton University Press, 2000, p. 304 .

31. "by blindly worship the West and a process of indiscriminate imitation", Wang Y. C., op. cit., p. 21. 
32. Jean Bourjade, Préface, in Yuan Chaucer (Zhuoying), La philosophie morale et politique de Mencius, op. cit., p. 9.

33. "Over the five hundred years of Western expansion and the creation of colleges and universities in colonized areas since the beginning of the sixteenth century, this belief became so strong as to make people doubt their own wisdom, when that wisdom was not articulated in Western educational institutions and languages.", Walter Mignolo, op. cit., p. 304.

34. Yann Philippe, op. cit., p. 10.

35. Ibid., p. 11 .

36. « Discours de Wang Chung-Hui », Annales Franco-Chinoises (AFC), N ${ }^{\circ}$, pp. 5-14.

37. Yann Philippe, op. cit., p. 11.

38. Shigemi Inaga, "Philosophy, Ethics, and Aesthetics in the Far-Eastern Cultural Sphere: Receptions of the Western Ideas and Reactions to the Western Cultural Hegemony", Questioning Oriental Aesthetics and Thinking Conflicting Visions of "Asia» Under the Colonial Empires, Inaga Shigemi (Dir.), Kyoto, International Research Center for Japanese Studies, 2011, p. 34.

39. Joël Thoraval, "De la philosophie en Chine à la "Chine" dans la philosophie ", Perspectives chinoises, $\mathrm{n}^{\circ} 4,1992$, p. 41.

40. Chen Lai, "Studying Chinese Philosophy: Turn-of-the-century's Challenges?", Michel Masson et Kao Chia Chi (Trad.), Revue Internationale de Philosophie, $n^{\circ} 2$, 2005, note p. 190.

41. Michael Lackner, «Les avatars de quelques termes philosophiques occidentaux dans la langue chinoise ", Études chinoises, vol. XII, n² 2, automne 1993, p. 138.

42. Ibid., p. 138.

43. Fu Yan, 厳復 (1854-1921) a été un fervent défenseur de l'occidentalisation de la Chine. Il est selon Cai Yuanpei "le premier à introduire la philosophie occidentale au dernier demi-siècle." (Cai Yuanpei, Wushinian lai zhongguo zhi zhexue五十年來 中國之哲學, 1985, p. 274.)

44. Shigemi Inaga, op. cit., p. 34.

45. Joël Thoraval, op. cit., note p. 54.

46. Ibid., p. 41.

47. Ibid., p. 49 .

48. Anibal Quijano, "Coloniality of Power and Eurocentrism in Latin America", International Sociology, Vol. 15, n², June 2000, pp. 217-234.

49. Walter Mignolo, series of lectures « The Spirit Returns to the East » at the Hong Kong Advanced Institute for Cross-Disciplinary Studies of City University of Hong Kong, http://www6.cityu.edu.hk/hkaics/activities/201203spirit/e_info.html (consulté le 29/03/2012).

50. Anibal Quijano, 'Raza', 'Etnia' y 'Nación' en Mariátegui : Cuestiones Abiertas' in Forgues, R. (ed.) José Carlos Mariátgui y Europa: El Otro Aspecto del Descubrimiento Peru: Empresa Editora Amauta S.A., 1993, p. 167-187 in Ramon Grosfoguel, «Les implications des altérités épistémiques dans la redéfinition du capitalisme global. Transmodernité, pensée frontalière et colonialité ", Anouk Devillé et Anne Vereecken (Trad.),Multitudes, 26(3), p. 57.

51. Ibid., p. 61.

52. Joël Thoraval, op. cit., p. 41.

53. Ibid.

54. Ibid.

55. Ibid., p. 42.

56. Michael Lackner, op. cit., p. 136.

57. Florent Villard, op. cit., p. 2.

58. Michael Lackner, op. cit., pp. 136-137.

59. 《袁振英投考北京大学的时候, 最初的选择是要进入西洋哲学系, 但当时的北 京大学只有中国哲学系, 他只好进入了西洋文学系》. [Yuan Zhenying a passé le concours pour entrer à l'Université de Pékin, son choix initial était d'entrer dans le département de philosophie occidentale, mais à l'époque l'Université de Pékin 
n'offrait qu'un département de philosophie chinoise, il a donc étudié la littérature occidentale], 袁振英——扑朔迷离的共产党员身份 (2013-05-02), 袁振英——扑朔迷 离的共产党员身份, 作者:家在桂林 http://blog.sina.com.cn/s/ blog_4cc1f6ea0101en8s.html (consulté le 13/01/2014).

60. Voir la lettre de Li Shizeng à Shen Pe-tsan (directeur de l'Institut) du 26 janvier 1932.

61. "L'amitié franco-chinoise", A. Vicard, in La vie lyonnaise, 3 années, n67, 10/12/1921, p. 22.

62. La thèse Huang Zengyue est une étude comparative sur les philosophies de Laozi, Kongzi, Mozi. Il y présente tour à tour la vie, l'œuvre des philosophes, leurs ressemblances et différences ainsi que leurs influences.

63. Lettre 28 mai 1925 Jean Bourjade à Maurice Courant, son directeur de thèse, (165-57 c).

64. English version: "the rest of the World was either the scene of interesting human achievements to study and understand", Walter Mignolo, op. cit., p. 304.

65. Voir Anne-Lise Dyck, « La Chine hors de la philosophie », in Anne Cheng (Dir.), « Y a-t-il une philosophie chinoise ? ", Extrême Orient - Extrême Occident, $n^{\circ} 27$, Saint-Denis, Presses Universitaires de Vincennes, 2005, pp. 13-47. Anne Cheng, Histoire intellectuelle de la Chine, Cours : Confucius revisité : textes anciens, nouveaux discours (suite),pp. 16-18. http://www.college-de-france.fr/media/annecheng/UPL66281_Cheng.pdf (consulté le 14/01/14).

66. Yang Jiaqing, "Anne Cheng : État des lieux de la Sinologie française contemporaine", http://french.beijingreview.com.cn/magazine/2010-11/05/ content_315007_2.htm, (consulté le 05/02/2014).

67. Walter Mignolo, The Darker Side of Western Modernity: Global Futures, Decolonial Options, Duke University Press, 2011, p. 81. Voir aussi Osamu Nishitani \& Naoki Sakai, Sekaishi no kaitai, (La Déconstruction de l'histoire mondiale), Tokyo, Ibunsha, 1999.

68. Naoki Sakai, La Théorie et l'Occident. Sur le problème de Humanitas et Anthropos, (Didier Renault Trad.), Transeuropéennes, 2 Août 2011, pp. 6-7. http:// www.transeuropeennes.eu/fr/articles/316/La_Theorie_et_l_Occident (consulté le 05/02/2014).

69. Joseph Needham souligne l'idée assez répandue que la langue chinoise comme langue "idéographique aurait été un puissant facteur de retard pour le développement d'une science moderne en Chine. » (Joseph Needham, La science chinoise et l'Occident, Trad. de l'anglais par Eugène Simon, Paris, Seuil, 1973, p. 38.) Cette idée trouve son origine chez les ethnologues comme Lucien Lévy-Bruhl qui au début du vingtième siècle, institue la langue primitive comme une langue simple. Il «a voulu prouver que les langues des peuples primitifs étaient dépourvues de mots abstraits ou génériques, d'où l'inaptitude de leurs locuteurs au raisonnement ou aux généralisations. » Marina Yaguello, Science et Avenir, 2000/12 -2001/1, n²125, p. 6.

70. «Cette abondance de lettres est à mon sens la source de l'ignorance des Chinois, parce qu'ils emploient toute leur vie à cette étude, \& qu'ils n'ont presque pas le temps de songer aux autres sciences, s'imaginant être assez savants quand ils savent lire. ", Louis Lecomte, Lettre VII à l'archevêque duc de Rheims, Nouveaux Mémoires sur l'État Présent de la Chine, Tome 1, Paris, Anisson, 1697, p. 307

71. Gottfried Wilhelm Leibniz, Discours sur la Théologie Naturelle des Chinois, Paris, L'Herne, 1987, p. 36.

72. Walter Mignolo, op. cit., p. 81.

73. Edmond Goblot in Huang Zengyue, op. cit., pp. VIII-IX.

74. Sophie Bessis, L'Occident et les autres: Histoire d'une suprématie, Paris, La Découverte, 2001, p. 315.

75. Ibid., p. 27.

76. Voir G. W. F.Hegel, La Raison dans l'Histoire : Introduction à la philosophie de l'histoire, Paris, Plon, 2000, p. 247. 
77. Jean Bourjade, Préface, in Yuan Zhuoying, op. cit., p. 7.

78. Edmond Goblot in Huang Zengyue, op. cit., p. X.

79. Ibid.

80. Sur la notion d'eurocentrisme, voir Jack Goody, The Theft of History, Cambridge, Cambridge University Press, 2007 où il soutient que l'Europe a "volé » des notions de l'Orient afin de se construire. Yves Citton « Vers une Europe postidentitaire ", Multitudes, n¹4, 2003/4, pp. 61-71, Samir Amin, Modernité, religion et démocratie : Critique de l'eurocentrisme, critique des culturalismes, Lyon, Parangon-Vs, 2008, Immanuel Wallerstein, "Eurocentrism and its Avatars: The Dilemmas of Social Science ", Keynote address at the ISA East Asian Regional Colloquium, "The Future of Sociology in East Asia ", 22-23 November 1996, Seoul, Korea, co-sponsored by the Korean Sociological Association and International Sociological Association, http://www.iwallerstein.com/wp-content/uploads/docs/ NLREURAV.PDF (consulté le 04/02/2014)

81. Edmond Goblot, Préface, in Huang Zengyue, op. cit., p. VIII.

82. Ibid., p. IX.

83. Ibid., p. XIV.

84. Huang Zengyue, op. cit., p. XV.

85. Ibid., p. 254.

86. Ibid., p. 257.

87. Ibid., p. 268.

88. Ibid.

89. Ibid.

90. P.-L. Couchoud, Sages et poètes d'Asie, Paris, 1916, p. 272.

91. Huang Zengyue, op. cit., p. 254. Il a également défendu les idées de démocratie et d'éducation pour les femmes, comme Cai Yuanpei. Il meurt en 1966 sous la répression de la Révolution culturelle, sa formation à Lyon fait partie de ses dix chefs d'accusation édictés par les jeunes gardes rouges : 2 《黄曾悑是外国帝国主义 培养的洋奴, 他崇洋媚外, 里通外国, 为特务机关提供情报。”[Huang Zengyue fut un serviteur des étrangers, formé par eux, il les vénérait, il s'est rendu chez eux et il a fourni des informations aux services secrets étrangers.] http:// llz1938.blog.163.com/blog/static/1138955142011102441837537/ (consulté le 10/01/2014)

92. "this was the location for the famous Lyons Sino-French Institute, which embodied a century of dreams and friendship between the two countries' intellectuals.” Duanmu Mei, “The Lyons Sino-French Institute”, 中外文化交流 : 英 文版, China \& The World Cultural Exchange, 中華人民共和國文化部, 中國對外交 化交流協會, 05/2012.

93. Voir Walter Mignolo, 'Géopolitique de la connaissance, colonialité du pouvoir et différence coloniale', op. cit..

94. Alain Badiou, "Jullien l'apostat ", in Oser construire : Pour François Jullien, Paris, Seuil, 2007, p. 140.

95. Lettre du Père Parrenin à M. Dortous de Mairan du 11 août 1730, in Charles Le Gobien, ed., Lettres édifiantes et curieuses, écrites des missions étrangères, Tome 12 (Lyon: J. Vernarel, 1819), 53.

96. Ibid., p. 55.

97. Ibid., p. 54.

98. Voir Huang Zengyue, op. cit., pp. 261-265.

99. Voir William J. Duiker, "Ts'ai Yuan-p'ei and the Confucian Heritage", Modern Asian Studies, Vol. 5, $\mathrm{n}^{\circ}$ 3, 1971.

100. A la fin de ses études en France, Yuan Zhuoying est retourné à Guangdong à l'école Nationale Normale Supérieure du Guangdong en tant que professeur, pour enseigner aux sections de troisième et quatrième années la philosophie et l'éthique. http://blog.sina.com.cn/s/blog_4cc1f6ea0101en8s.html (consulté le 13/01/2014).

101. Yuan Zhuoying, op. cit., p. 125.

102. Ibid. 
103. Ibid., pp. 129-130.

104. Ibid., p. 130.

105. Ibid., p. 131.

106. Ibid., p. 205.

107. Jean Bourjade in Yuan Zhuoying, op. cit., p. 7.

108. Ibid., p. 10.

109. Voir la Lettre du Père Parennin à Mr Dortous de Mairan, directeur de l'Académie des sciences du 11 août 1730, in Charles Le Gobien (Dir.)., Lettres édifiantes et curieuses, Tome 12, Lyon, J. Vernarel, 1819 p. 52.Voir Anne-Lise Dyck, op. cit., pp. 17-26. Cette idée est défendue au XIX ${ }^{\mathrm{e}}$ siècle notamment par Jean-Marie De Gérando, Histoire comparée des systèmes de philosophie, Paris, 1822, Tome 1, Chapitre III, p. 199, Hegel, Leçons sur l'histoire de la philosophie, Paris, Gallimard, 1964, p. 77.

110. Ibid., p. 9.

111. Voir François Jullien, Chemin faisant, connaître la Chine, relancer la philosophie, Paris, Seuil, 2007, François Jullien et Thierry Marchaisse, Penser d'un dehors (la Chine), Entretiens d'extrême Occident, Paris, Seuil, 2000 et François Jullien, Un sage est sans idée ou l'autre de la philosophie, Paris, Seuil, 1998. L'Orientalisme est exprimé par le fait que la pensée chinoise est relayée au musée par cette mise à distance, mystifiée et hermétique, réduite à un faire-valoir et utilisée pour revenir à un eurocentrisme puisqu'il s'agit de l'utiliser telle une "prothèse mentale" afin de mieux comprendre la pensée européenne et combler ses lacunes.

112. Jean Bourjade in Yuan Zhuoying, op. cit., p. 9.

113. Ibid., p. 10.

114. Edmond Goblot in Huang Zhengyue, op. cit., p. X.

\section{RÉSUMÉS}

Bien que beaucoup d'études aient été menées concernant les étudiants chinois partis étudier la philosophie à l'étranger au début du XX $\mathrm{XX}^{\mathrm{e}}$ siècle (tel Hu Shi aux Etats-Unis), encore trop peu de travaux se sont penchées sur les étudiants qui se sont formés en philosophie en France et notamment à l'Institut Franco-chinois de Lyon. Cet article, aidé des théories de l'orientalisme et $\mathrm{du}$ postcolonialisme, se demande si la géopolitique du savoir philosophique, en tant que domination intellectuelle de l'Occident sur le reste du monde, s'est manifestée dans le contexte de l'Institut Franco-chinois de Lyon, et si oui comment. À travers l'analyse des paratextes et des thèses de deux étudiants en philosophie de l'Institut Franco-chinois de Lyon, cette étude soutient que cette institution est un produit des effets de la géopolitique du savoir intellectuel où le traitement des philosophies occidentales et chinoises suit le découpage moderne/colonial du savoir. On y trouve l'illustration du déplacement de la Chine en tant qu'objet de savoir (anthropos) étudiée par les producteurs du savoir (humanitas), découplage instituant la Chine en dehors de la philosophie. 


\section{AUTEUR}

\section{MARIE-JULIE MAITRE}

Marie-Julie Maître est professeure adjoint de littérature et de philosophie française au département de français de l'Université Tamkang (Taïwan) depuis Août 2012. Elle fut chercheuse au Département des Études Asiatiques et Internationales ainsi qu'au Hong Kong Advanced Institute for Cross-Disciplinary Studies à City University de Hong Kong entre 2010 et 2012. Son doctorat en philosophie, achevé en 2010, étudie la circulation des idées et théories, notamment la réception et la représentation de la philosophie chinoise en France du XVI ${ }^{\mathrm{e}}$ au XXI ${ }^{\mathrm{e}}$ siècle. 